\title{
An Approach for Narrow Band Interference Detection in Satellite Communication Using Morphological Filter
}

\author{
Jing $\mathrm{Hu}^{1}{ }^{1}$ a, Dongming Bian ${ }^{1}$, Zhidong Xie ${ }^{1}$, Yongqiang $\mathrm{Li}^{1}$, Lehao Fan ${ }^{2, \mathrm{~b}}$ * \\ ${ }^{1}$ Institute of Communication Engineering, PLA University of Science and Technology, \\ Nanjing 210007, China \\ 2 Design Ltd. of JiangSu Posts and Telecommunications Planning Academy, Nanjing 210006, \\ China \\ axiaoxiaomissy@hotmail.com, b106001318@qq.com
}

\begin{abstract}
Keywords: Satellite communication; Morphology; Gradient; Narrow band interference Detection; Noise Floor

Abstract. How to precisely detect co-channel narrow band interferences using single threshold is a challenge for satellite transponder spectrum monitor, especially in the condition of many signals which parameters are different. A new method for interference detection in satellite communication is proposed based on mathematical morphology. The paper uses an improved morphological gradient filter which belongs to image and graphics filed to deal with the spectrum data that is viewed as one-dimensional gray-scale image, and then search the gradient of interference edges to detect the position of it. Simulation results show that the proposed method can detect interference at one time when there are different signals with interference; it has not effect by the non-flat noise floor and has low computational complexity, is suitable for real spectrum monitor in satellite communication system
\end{abstract}

\section{Introduction}

In satellite communication, there are many co-channel interferences that come from the ground wireless communication system, other satellite communication system or some radar signals. How to effectively monitor the co-channel interferences is prerequisite for eliminating it and is basic to improve the system efficiency, so the interference monitor is becoming one of the important issue in satellite communication research filed ${ }^{[1]-[4] .}$

The interference monitor and suppression in different communication systems has attracted many attentions all around the world. Paper [5]-[8] introduce some technologies of narrow band interference suppression in spread spectrum communication system, paper [8] use a new transform selective mode distinguish technique to detect interferences of different types to solve the problem that traditional detection techniques could only detect partial common interferences and attend by high computation complexity. Paper [3][4] does not use a standard threshold, but use a threshold similar to the signal power spectrum to detect the co-channel interference signal, it can improve the detection ratio of small interference signals, but the method need to find out the modulation type of signal firstly and not suitable for environment of more than one signals. The paper introduce mathematical morphology to interference detection in satellite communication, firstly the spectrum data is viewed as one-dimensional gray-scale image, and then search the gradient of interference edges by morphological filter to detect the position of it. The proposed method can detect interference at one time when there are different signals with interference and has low computational complexity, is practical for real spectrum monitor in satellite communication system.

This paper mainly contains the following sections, section 2 introduces the application of theory of morphological filer in the of communication signal processing, section 3 discusses the application of morphological filter to detect the co-channel interferences, and section 4 illustrates the validity and feasibility of the proposed interference detection performance. 


\section{Rationale of Morphological Filter}

The techniques of mathematical morphology are based on set theory, integral geometry, convex analysis and topology. It is a set-theoretical methodology for image analysis, can rigorously capture many aspects of the geometrical structure of signals in a way that is consistent with human intuition and perception ${ }^{[9][10]}$. Mathematical morphology has been widely used for biomedical and electron microscopy image analysis, and has recently become a topic of interest for academic research in the field of communication signal processing ${ }^{[11]}$

The fundamental operators of mathematical morphology are dilation and erosion, which are defined as in (1) and (2):

$$
\begin{aligned}
& \boldsymbol{X} \oplus \boldsymbol{B}=\mathrm{U}_{b \in \boldsymbol{B}} \boldsymbol{X}+b=\{x+b: x \in \boldsymbol{X}, \mathrm{b} \in \boldsymbol{B}\} \\
& \boldsymbol{X} \Theta \boldsymbol{B}=\mathrm{I}_{b \in \boldsymbol{B}} \boldsymbol{X}-b=\{z: \boldsymbol{B}+z \in \boldsymbol{X}\}
\end{aligned}
$$

$\mathrm{X}$ represents the set of input signal. $\mathrm{B}$ is a compact set of small size and simple shape, called a structuring element.

The one widely used for purposes is a rectangular shape consisting of $\mathrm{N}$ pixels. Dilation means that moving structure element B over signal space, when the structure element B absolutely overlaps part of signal, adds up the values of each element of structure element B and signal that overlapped, and then chooses the max value of the sum to replace the fixed point. To erode the signal X means that the structure element B moved over the signal X to search these shaded points which can placed B fitly.

It is widely use of simple structure of structure element B in communication signal processing. The purpose of the paper is to monitor the interferences in satellite communication system using morphological filter, the filter processing is only pay attention on the change of horizontal points, so the flat structure element $\mathrm{B}$ is a better choice. The following simulation of the paper uses flat structure element B.

\section{Narrow Band Interference Detection Based on Morphological Filter}

\section{The Model of Narrow Band Interference In Satellite Communication System}

In satellite communication system, the co-channel interference is one of main reasons of communication quality reducing. There are mainly two kinds of co-channel interference, which are narrow band interference and wide band interference that occupy different transponder width. For narrow band interference is more common, so the paper focuses on narrow band interference detection. Suppose the received detection signal is represented by $y(t)$, as in (3),

$$
y(t)=\sum_{i=1}^{N_{s}} s_{i}(t)+n(t)+\sum_{k=1}^{N_{J}} j_{k}(t)
$$

$s_{i}(t)$ represents the narrow signals, $N_{s}$ is the number of narrow signals, $j_{k}(t)$ represents narrow band interference, $N_{J}$ is the number of narrow band interference, and $n(t)$ represents the noise. It can be prove that there is no correlativity in narrow signals, interference and noise. So the power spectrum of received detection signals $y(t)$ is the sum of power spectrum of each part. The fig. 1 shows the power spectrum model of one received signal that has one co-channel narrow band interference. The interference detection is always include two steps, firstly judge whether there are interference and secondly find out the parameters of interference. The paper proposed procedure can complete the two steps at once using morphological filter.

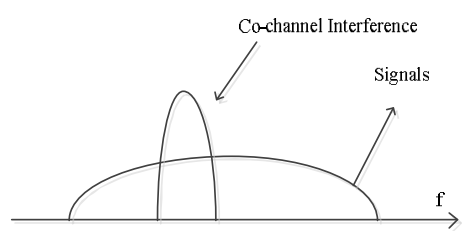

Fig. 1.Model of co-channel narrow band interference 


\section{Rationale of Narrow Band Interference Monitor Using Morphological Filter}

Morphological filter is a method that is the transform or combination of mathematical morphology to achieve the useful information from signals, and it is a non-linear signal process of modifying geometry character of multi-dimensional signal.

The morphological gradient, which is a filter method of basic morphological operation combination, definition as in (4), $\boldsymbol{X}$ is detected signal and $\boldsymbol{B}$ is flat structure element.

$$
G(\boldsymbol{X})=(\boldsymbol{X} \oplus \boldsymbol{B})-(\boldsymbol{X} \Theta \boldsymbol{B})=\bigcup_{b \in \boldsymbol{B}} \boldsymbol{X}+b-\mathrm{I}_{b \in \boldsymbol{B}} \boldsymbol{X}-b=\{x+b: x \in \boldsymbol{X}, \mathrm{b} \in \boldsymbol{B}\}+\{z: \boldsymbol{B}+z \in \boldsymbol{X}\}
$$

Interference model is defined as I in fig. 2(a), deal with it by morphological gradient filter using the flat structure element as B. The fig. 2 (b) shows the change process of both sides of interference I that filtered by morphological gradient filter. There are two peaks in the position of interference sides, and then it is easy to fix the position of interference I through choice suitable threshold to find them.

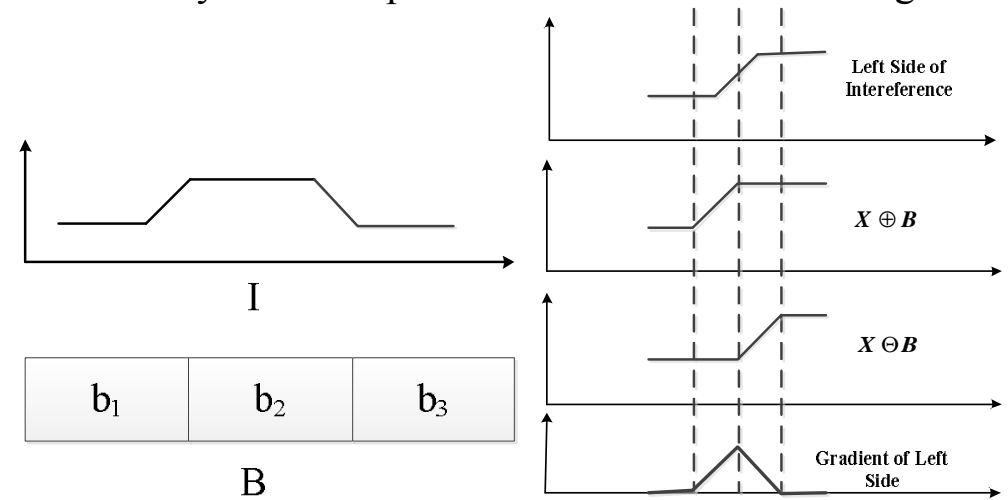

(a)

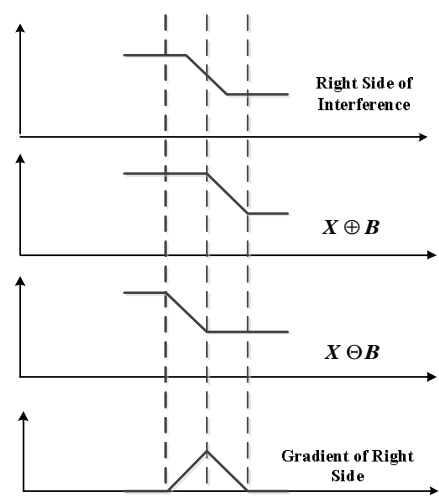

(b)

Fig. 2. Gradients of Interference both Sides

\section{Method of Narrow Band Interference Monitor Using Morphological Filter}

Interference detection is the basic for interference analysis and interference cancellation. Based on introduce of morphological gradient filter theory, it is clearly that the method can be used to detect the position of narrow band interference. The paper proposed method includes four modules: spectrum smooth, calculate gradient, find local position of maximum (minimum) and judge interference. Generally at last there is a statistical process to enhance the veracity of interference detection, which based on the character of signal instantaneous spectrum. Now introduce the four modules in details.

1) Smooth

The purpose of smooth is to reduce the noise influence on follow steps. Deal the spectrum after smoothing with morphological gradient filter, the gradient values of interference edges are higher than noise part, and then it is easy to choice suitable threshold to distinguish interference from noise. .

2) Calculate gradient

From the formula (4), it is obviously that gradient is the minus of dilation and erosion, specific as follow steps:

Step 1: choice the flat structure element B of length $L$, as in (5),

$$
B=b_{0}, b_{1}, \mathrm{~L}, b_{L-1}
$$

$\mathrm{L}$ is the nature number that is smaller than the length of spectrum data, and $b_{i}$ is the random real number of the i-th data of B.

Step 2: Choose one object point of frequency spectrum data to analysis, and then with the beginning of the object point get some 'analyzing data' of frequency spectrum, which is the same length of structure element B.

Step3: To get the erosion result of object point through the 'analyzing data' erosion by structure element $\mathrm{B}$ as formulation (2); and get the dilation result of object point through the 'analyzing data 'dilation by structure element B as formulation (1);

Step4: The gradient value of object point is the minus of the erosion result and dilation result of it; 
Step5: Modified gradient values according to the trend of frequency spectrum data change. If the point of frequency spectrum data is in ascending border, the gradient of the point keep positive, but if the point is in descending border, the gradient of point is change to negative.

3) Set the threshold to find out the position of local maximum(minimum) of the gradient.

There are non-zero gradient values of points that has ascending or descending change trend .For the smooth frequency spectrum data, the absolute value of gradient amplitude of interference is higher than noise's, so it is easy to fix on the position of interference through threshold setting. Figure 5 shows that the red broken lines are threshold, it is easy to distinguish the gradient of noise from others through the threshold line.

4) Narrow interference determinant

The both sides of narrow interference would be present positive and negative local peaks through the processes referred before, and the paper proposed the method that combines the points of the two kinds of peaks to determinate the position of interference, which is described in details as follows.
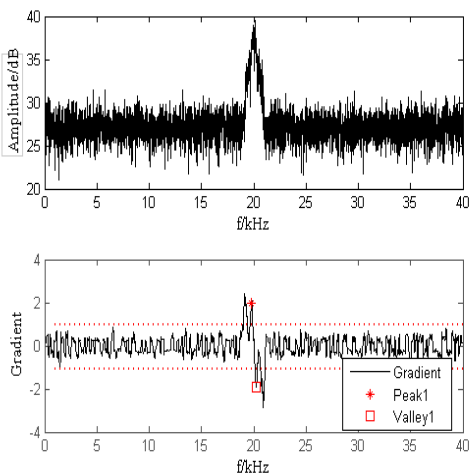

(a)
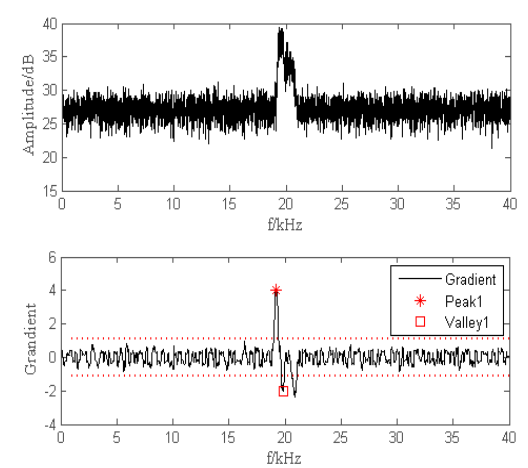

(b)
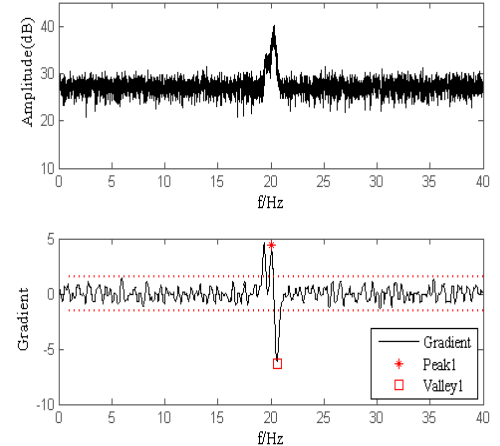

(c)

Fig. 3. Spectrum of Interference in One Signal and Gradients

According to the relative position of signal and narrow band interference, it can be divided into two cases, the one is there is no side of narrow band interference and signal is overlapped as upside of figure 3(a) shows, and the other is opposite that one side of them is overlapped as upside of figure 3 (b) (c) show. For the first case, there is a pair of positive and negative peaks in a signal bandwidth as shown in downside of fig. 3(a), it is easy to monitor the narrow band interference through determinate the two peaks positions. For the second case, there is one side of interference that is overlapped with signal side, so the gradient of narrow band interference only presents one peak in signal bandwidth as shown in fig. 3 (b) and (c), the position of interference is fixed by the signal side and the negative (opposite) peak.

\section{Simulation and performance analysis}

Simulation operates in low frequency band, the carry frequency is $f_{c}=20 \mathrm{kHz}$, and the method also can be used in higher frequency band.

The upside of fig. 4 shows there are four BPSK Signals in $40 \mathrm{KHz}$ monitor bandwidth, that the second ,third and fourth signals are interfered by co-channel narrow band interference especially two interferences in the third signal. The noise floor is flat. The $R_{b}$ of signals are $2 \mathrm{ksps} 、 1 \mathrm{ksps}, 4 \mathrm{ksps}$ and $0.5 \mathrm{ksps}$ from left to right, and $E_{b} / n_{0}$ of them are $9 \mathrm{~dB} 、 7 \mathrm{~dB} 、 3 \mathrm{~dB}$ and $5 \mathrm{~dB}$. The JNR of interference are $7 \mathrm{~dB} 、 6 \mathrm{~dB} 、 6 \mathrm{~dB}$ and $9 \mathrm{~dB}$. The downside of fig. 5 shows the gradient result of monitor bandwidth using the method that the paper proposed. It is obviously that there are local positive and negative peaks of narrow band interference both sides position, as marked by the red broken line. Using the threshold can determinate these local peaks, and then match the positive and negative peaks as referred before to monitor the interferences position at once. 

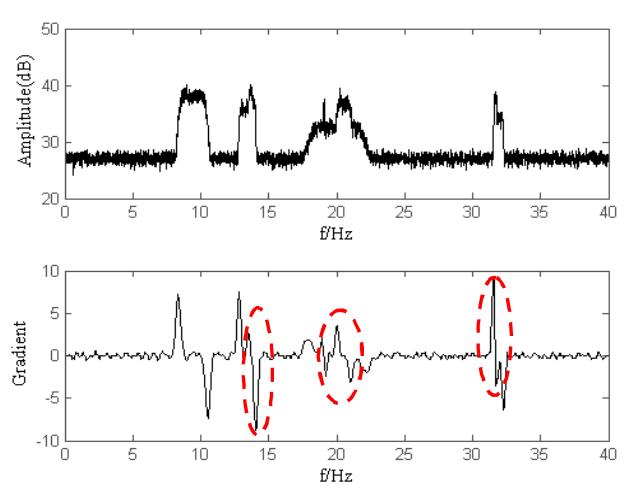

Fig . 4. Spectrum of Interference in Signals

With Flat Noise Floor and Gradients
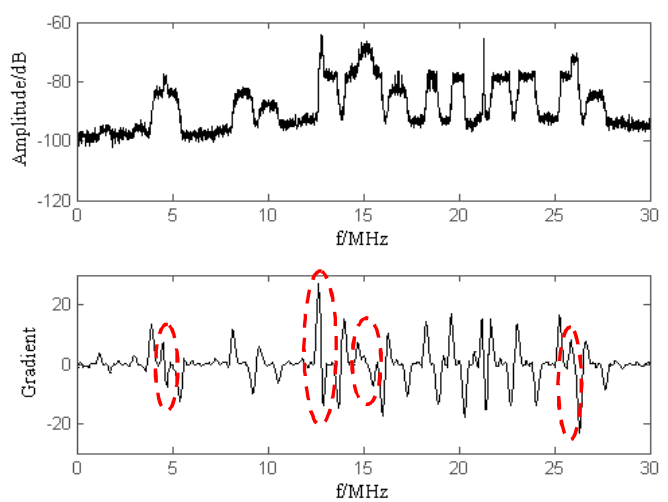

Fig. 5. Spectrum of Interference in Signals with varying Noise Floor and Gradients

Fig. 5 upside is $30 \mathrm{MHz}$ bandwidth frequency spectrum of some satellite in $\mathrm{Ku}$ span that the narrow band interferences have been added to it. The noise floor of this spectrum band is varying. There are many different signals in the bandwidth, and the first, fourth, fifth and twelfth signal has been add co-channel narrow band interference whose parameters has been descripted in table1. The spectrum has been processed by the method paper proposed and the fig 5 downside is the gradient result of it. It is obviously that there are local positive and negative peaks in the position of narrow band interference both sides marked by red broken line. Matching the positive and negative peaks as referred before can determinate the interferences position at once. It is good choice to synthesize more than one result to promote veracity and precision.

TABLE I. Parameters of Interference

\begin{tabular}{|c|c|c|c|c|}
\hline Number & $\begin{array}{c}\text { Fc of Interference } \\
(\mathbf{M H z})\end{array}$ & $\begin{array}{c}\text { JNR(dB } \\
\text { ) }\end{array}$ & $\begin{array}{c}\text { Rb of } \\
\text { Interference(kbps) }\end{array}$ & $\begin{array}{c}\text { Modulation } \\
\text { Type }\end{array}$ \\
\hline 1 & 4.62 & 3 & 0.2 & BPSK \\
\hline 2 & 12.78 & 10 & 0.25 & BPSK \\
\hline 3 & 15.13 & 5 & 0.4 & BPSK \\
\hline 4 & 26.10 & 4 & 0.8 & BPSK \\
\hline
\end{tabular}

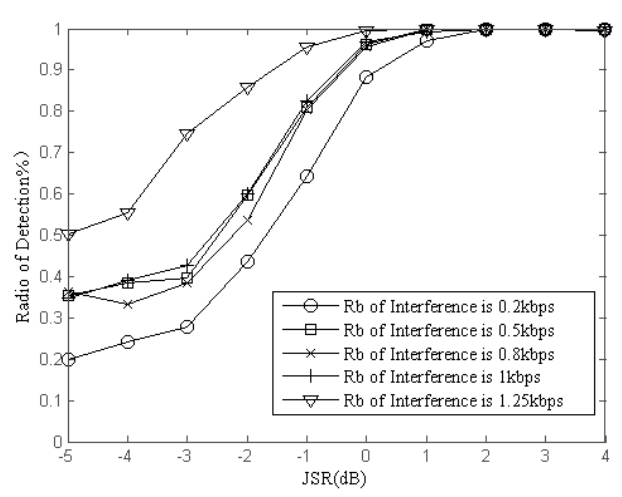

(a) Rb of Signal is $4 \mathrm{kbps}$

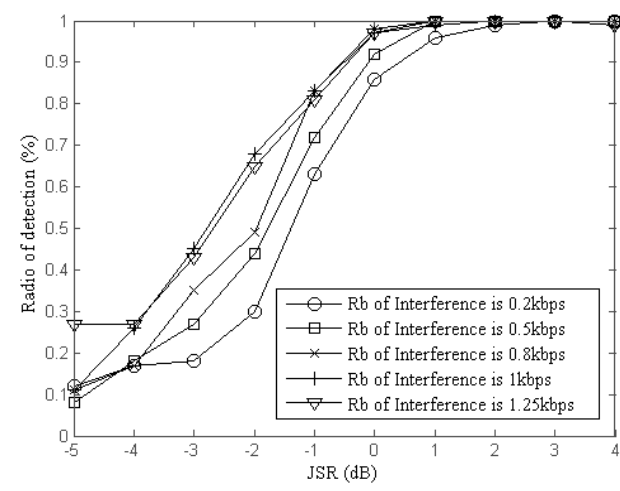

(b) Rb of Signal is $2 \mathrm{kbps}$

Fig.6. Radio of Detection in Different JSR

Figure 6 shows that when there is only one signal and one narrow band interference the detect probability of interferences that have different bit rate and JSR. The bit rate of signals are $4 \mathrm{kbps}$ and $2 \mathrm{kbps}, f_{s}=80 \mathrm{kHz}, E_{b} / n_{0}=3 d B$ and number of FFT is 8192 .

It is clearly that if the narrow band interference has the same bit rate, the ratio of detect increases with the JSR growth, and in the condition of the same JSR the interference that has higher bit rate has higher detect ratio. In fig. 6 (a) and (b) the bit rate of signals are different, but for the same narrow band interference the change trend is resemble, and when the JSR is $1 \mathrm{~dB}$ the detect ratio is exceed $90 \%$. 


\section{Conclusion}

The morphological gradient detect method paper proposed can be used to monitor multiple interferences when there are more than one signals in satellite transponder which has non-flat noise floor. The procedures of this method are linear processes; it is suitable for hardware implementation for low complexity and calculation cost that is proportional to the data length.

\section{Acknowledgement}

The research work was supported by National Natural Science Foundation of China under Grant No. 91338201 , 91438109, 61401507 and National High Technology Research and Development Program of China (“863” Program) (2012AA01A510).

\section{References}

[1] Kaitsuka T, Inoue T. Interference cancellation system for satellite communication earth station[J]. IEEE Trans Commun, 1984,7(7):796-803.

[2] Sugiyama T, Kubora S, Morikura M, et al. A coded VSB-QPBK transmission scheme for digital satellite communications in co-channel interference enviroments[J]. IEEE trans Aerosp\&Electron Syst , $1996,7(3): 1174-1181$.

[3] Sun Liping, Hu Guangrui, Wu Jun. A New Transform Domain Method for Narrow Band Interference Detection in DQPSK Satellite Communication Systems.[J] Journal of Data Acquisition \& Processing. 2003.06,18(2), 132-135 (In Chinese).

[4] Wu Jun, Hu Guangrui, Sun Liping, An FFT Narrow-Band Interference Detection Scheme in Digital Satellite Communication Systems.[J] Journal of ShangHai JiaoTong University.2003.10(37), 1600-1602 (In Chinese).

[5] Ho K C, Lu Xiaoning, Mehta V. Adaptive blind narrowband interference cancellation for multi-user detection[J]. IEEE Trans, on Wireless Communication,2007, 6(3):1024-1033.

[6] BuzziS, Antonio D M. Code-aided blind detection of the transmission rate for multirate direct-sequence CDMA systems[J].IEEE Trans. on Signal, 2005,53(6):2182-2192;

[7] Fu Weihong, Song Changhan, Huang Kun. Narrow-band Interference Suppression in Transform Domain Based on Difference-cluster-threshold Algorithm.[J] Journal of Electronics \& Information Technology, 2013,35(12): 2960-2965 (In Chinese).

[8] Yin Fulian, Guo Lili, Lu Man-hong, Research on common interference detection techniques of spread spectrum TT\&C systems[J]. Systems Engineering and Electronics, 2009.09,31(9), 2195-2199 (In Chinese).

[9] Gao Hengzhen, Wan Jianwei, Nian Yongjian, etal. Hyperspectral Image Classification Algorithm Based on Spectral-spatial Hybrid Features and SVM [J]. Journal of Astronautics, 2011, 32(4): 917-921 (In Chinese).

[10]Kong Yingying, Zhou Jianhong, Zhang Miao.SAR Image Restoration Bsed on GAbbis Markov Random Field Model and Connected Clustering.[J]. Acta Aeronoutica et Astronautica Sinica, 2010, 31(2): 310-317 (In Chinese).

[11] Wang Yongming, Zhang Eryang, Zhao Jinli. Signal detection in broadband reconnaissance receiver based on morphological filter [J]. Journal of Applied Sciences, 2009, 27(4): 343-347 (In Chinese). 